\title{
THE EFFECTS OF COGNITIVE BEHAVIORAL INTERVENTIONS ON REMOVING BARRIERS TO TREATMENT ADHERENCE IN HEMODIALYSIS PATIENTS
}

\author{
MITRA ZOLFAGHARI ${ }^{1}$, FATEMEH SOOKHAK ${ }^{2 *}$, SEYYED HANNAN KASHFI ${ }^{3}$, EGHBAL SEKHAVATI ${ }^{2}$, REZA TABRIZI ${ }^{4}$
}

${ }^{1}$ Department of E-learning in Medical Education, Nursing and Midwifery Care Research Center, Virtual School, Tehran University of Medical Science, Tehran, Iran. ${ }^{2}$ Department of Nursing, Larestan School of Medical Sciences, Larestan, Iran. ${ }^{3}$ Department of Nursing, Faculty Member of Larestan Nursing School, Larestan School of Medical Sciences, Larestan, Iran. ${ }^{4}$ Department of Health Policy, Health Policy Research Center, Shiraz University of Medical Sciences, Shiraz, Iran. Email: sookhakfatemeh@yahoo.com

Received: 12 January 2016, Revised and Accepted: 20 January 2016

\section{ABSTRACT}

Objective: Treatment adherence in chronic patients results in favorable treatment outcomes. Today, one of the main causes of mortality in hemodialysis patients is that of lack of treatment adherence. Identifying barriers to adherence to treatment is the first step to help these patients. The purpose of this study is to determine the effects of cognitive behavioral interventions on removing barriers to treatment adherence in hemodialysis patients.

Methods: This clinical study was carried out in the hemodialysis wards of Imam Reza Hospital of Larand Vali-e-Asr Hospital of Lamerd. The sample included 70 patients who were randomly assigned into two groups of intervention ( $n=35$ for even days)and control ( $n=35$ for odd days). The intervention group received a six-step cognitive behavioral treatment. The level of barriers to treatment adherence was assessed using a self-report questionnaire in two stages (pre-intervention and post-intervention). Data were analyzed using SPSS via independent t-test.

Results: Before the intervention, the two groups were not significantly different in terms of barriers to treatment adherence ( $p=0.68$ ). However, after the treatment regimen, the barriers significantly decreased for the intervention group. There was a significant difference between the two groups in terms of barriers to treatment adherence $(\mathrm{p}<0.001)$.

Conclusion: Given the efficacy of cognitive behavioral intervention, it can be used to identify barriers to adherence and design individualized education programs based on barriers to adherence in hemodialysis patients to increase their treatment adherence.

Keywords: Cognitive behavioral, Barriers to treatment adherence, Hemodialysis patients.

(C) 2017 The Authors. Published by Innovare Academic Sciences Pvt Ltd. This is an open access article under the CC BY license (http://creativecommons. org/licenses/by/4. 0/) DOI: http://dx.doi.org/10.22159/ajpcr.2017.v10i1.10689

\section{INTRODUCTION}

The increasing prevalence of chronic diseases is one of the challenging issues' communities and health staff face. One of these diseases that create considerable tensions for patients is end-stage renal disease [1] Kidney failure is a life-threatening condition, and annually millions of people worldwide suffer from this disease. A lot of money is spent by health systems to prevent and treat the disease. A high incidence of 1000 cases per million population in developed countries is expected [2]. For example, in the United States, the incidence and prevalence of kidney failure is increasing, so that the number of kidney transplant or dialysis patients increased from 340,000 in 1998 to 651,000 in 2010 [3]. Hemodialysis is the most common treatment used for these patients [1].

Several factors are known to affect dialysis adequacy including the treatment regimen. Adherence to treatment regimen includes the content of individual behavior to get drugs, following diets, lifestyle changes, and adherence to health-care guidelines. Correct management of hemodialysis patients is critical in terms of their adherence to treatment regimens and reduces symptoms such as muscle cramps, malnutrition, and infection [4]. Treatment non-adherence is a significant problem in patients with chronic diseases, including patients undergoing hemodialysis [5]. Hemodialysis patients have many problems in terms of treatment adherence due to their unique and complex treatment regimens. More than $50 \%$ of hemodialysis patients do not adhere to their treatment regimen [6].

Various factors, including patients' information on their diet, economic and social status, personal health beliefs, attitude toward treatment, and cultural differences can affect the treatment adherence in hemodialysis patients. Treatment non-adherence aggravates the disease, increases referrals to the hospital and hospitalization, and increases costs [7].

The first step to help chronically ill patients is to identify treatment barriers [8]. Perceived barriers refer to beliefs about the true costs of the new behavior. The person may believe that the new treatment is effective in reducing the severity of disease or may think that it is expensive, difficult, unpleasant, painful, or disturbing [9]. Identifying factors affecting treatment non-adherence is very important for health-care providers and enables them to use interventions to improve patients' adherence. Interventional strategies such as removing barriers to treatment adherence, patient education, and cognitive behavioral strategies can be used to improve treatment adherence in hemodialysis patients [10]. Beraz et al. stated that educational courses for patients undergoing hemodialysis can reduce problems associated with laboratory parameters diet compliance [11]. Sharp et al. found that psychological interventions improve adherence to hemodialysis fluid restrictions [12]. Developing strategies to improve treatment adherence is one of the goals of the treatment team. These strategies include cognitive behavioral and multimodal interventions. Cognitive behavioral treatments help patients understand their cognitive problems and change them. These changes can lead to changes in behavior [13]. Establishing communication between team members and patients motivates patients for treatment adherence and lets the treatment team increase patient satisfaction with their participation in the care program [5]. Given the importance of treatment adherence in hemodialysis patients, few studies were investigated the issue for these patients. In addition, to the best of our knowledge, this study 
was the first cognitive behavioral interventions that have been used in Iran to improve adherence in hemodialysis patients. Therefore, the purpose of this study was to investigate the effects of cognitive behavioral interventions on removing barriers to treatment adherence in hemodialysis patients.

\section{METHODS}

This study is a randomized clinical trial carried out between February 2013 and June 2013 in the hemodialysis wards of Imam Reza Hospital of Lar and Vali-e-Asr Hospital of Lamerd. Because of insufficient numbers of patients in a hospital, two hospitals that were homogeneous in terms of staff personnel, hemodialysis method, and the attending patients were selected. To prevent disclosure of patients' information, they were randomly assigned into two groups of intervention ( $n=35$ for even days) and control ( $n=35$ for odd days). In this study, based on Kakudate et al. (2009) study, to determine the sample size at a confidence level of $95 \%$ and power of $80 \%$, assuming that the effect size of cognitive behavioral therapy on treatment adherence should be at least $d=10$ so that the effect of the intervention can be considered statistically significant; the formula showed a sample size of 32 patients per group. Taking into account the sample loss, a sample size of 35 patients in each group was calculated; therefore, the total sample size was 70 patients.

Based on inclusion criteria, 20-60-year-old hemodialysis patients undergoing hemodialysis for at least a year 2 or 3 times/week in 3-4 hrs hemodialysis sessions were selected. They were capable of reading and writing. All participants were in poor conditions in terms of treatment adherence. This means that their scores on each component of the treatment adherence were $<50 \%$. Therefore, if the individual's scores on the self-reported treatment adherence questionnaire (20 questions) in each component (diet, medication, and restricted fluid intake) or the total score was $<50 \%$, the participant was considered as having poor treatment adherence or treatment non-adherence status and was included in the sample. It should be noted that the total score of the questionnaires was 44 calculated based on 100 (score of more than $75 \%$ indicates good adherence, $50-75 \%$ indicates a relatively good treatment adherence, and $<50 \%$ indicates poor treatment adherence).

Patients who for whatever reason did not wish to continue participating in the study or did not participate in any of the stages of intervention were excluded from the study. Convenience sampling was used. People referring to Hemodialysis unit completed treatment adherence questionnaire and those having non-adherence were identified and signed informed consent forms. The participants then were assigned randomly into two interventions and control groups (odd and even days). The treatment adherence barriers questionnaire (TABQ) is a self-report questionnaire developed by the researchers. TABQ was administered to both groups. The questionnaire included two parts.

The first part of TABQ tested demographic data and comprised two parts: (a) Questions about demographic data, life situation, education level, insurance status, income, and the caregiver and (b) information about the disease, duration of diagnosis, underlying causes of disease, and dialysis schedule.

The second part of TABQ included 15 questions in relation to socioeconomic variables, the patients' health beliefs and understanding of the disease and therapeutic regimens, false beliefs about the use and effectiveness of the treatment regimen, and intentional and unintentional forgetting to get recommended medicines and foods. Questions were scored based on a Likert rating on a range from 0 to 4 . In total, 15 questions had 60 points.

To determine the scientific validity of the questionnaire, content validity was determined. After reviewing books, new and relevant scientific literature, relevant research sites, and obtaining comments by the respected supervisor and advisors, the instrument was developed. Then, to determine face and content validity, it was sent to 10 faculty members of Tehran University of Medical Sciences. After collecting their ideas, their comments and proposed modifications were applied under supervision of the respected supervisor and advisors. The testretest method was used to determine reliability. The questionnaire was administrated to thirty qualified people and their scores were calculated. After just 2 weeks, the questionnaire was re-administrated to the same group. The Pearson correlation coefficient of 0.92 indicated an acceptable reliability level.

The intervention group received the six-step cognitive behavioral intervention adapted from Albright and Farquhar (1992) [14]. The method includes six steps. In step 1, identifying the problem, the barriers to treatment adherence were identified using a structured faceto-face interview and TABQ (questions such as: Why don't you adhere to your treatment regimen? what is the greatest barrier to your treatment adherence, etc. were asked). Step 2 involves creating confidence and commitment in the patient to correct inappropriate behavior. At this stage, the researcher helped the patient to understand problems and obstacles related to their non-adherence. Then, the patient and the researcher signed a contract to improve adherence. This was done by increasing the patients' motivation to change behavior. The researcher stated positive statements to increase the patients' confidence and interest in changing behavior. The patients were asked at the end of the session to write a diary including all the things they did on a daily basis to improve adherence until next session. In the 2 nd week, step 3 of the intervention, increasing awareness of behavior, was carried out. All of the patients' daily reports and their self-reporting statements regarding treatment adherence were reviewed and summarized. In step 4, training pamphlets were distributed to individual participants. The pamphlets include training related to treatment adherence in hemodialysis patients and had been designed based on barriers to treatment adherence. In the 4 th week of intervention, step 5 and 6 were carried out. In fact, the extent to which the program was effective on removing barriers to treatment adherence was evaluated. In case of the effectiveness of the program to change behavior, the researcher encouraged the patient to maintain the behavior change via motivational statements. These steps were conducted through structured face-to-face interviews in four 30 40 minutes sessions. Then, 3 months after the intervention, TABQ were completed again by the participants.

The control group did not receive any intervention after completing the questionnaire for the first time. They completed the questionnaire again 3 months later. Data were analyzed by independent t-test using SPSS version 16. It should be noted that the control group received routine care including receiving an educational pamphlet given to each patient at the time of entry into the unit and answering questions by staff. At the end of the study, the sample received the pamphlet containing educational materials in relation to the three areas of diet, medication, and fluid restriction.

Ethical issues in the research such as obtaining informed consent from the study individuals, informing the patients of their right to discontinue their participation freely and unconditionally at any stage of the investigation, obtaining permission from the university ethics committee, registration of the project at Iranian Registry of Clinical Trials and safeguarding the collection and analysis of data were considered carefully in this study.

\section{RESULTS}

During the study, 35 patients were assigned to each group. By the end of the study period, four patients were excluded from the study. A member of the intervention group did not participate in a step of the intervention and three members of the control group did not wish to continue participating in the study and were excluded. The mean \pm standard deviation age of patients was $51.02 \pm 13.58$ for the control group and $50.11 \pm 9.28$ for the intervention group. Other demographic characteristics are presented in Table 1 . It should be noted that the two groups were homogeneous in terms of demographic variables. The most frequent cause of dialysis was hypertension in the control group 
(37.2\%) and intervention group (48.6\%). Most patients in the control group (74.3\%) and intervention (74.3\%) had been undergoing dialysis for $1-5$ years.

As shown in Tables 2 and 3, treatment adherence was determined after completing the questionnaire by participants and analyzing the data. The level of treatment adherence before intervention was poor for most participants in the intervention group (83.9\%) and the

Table 1: Demographic characteristics of hemodialysis patients

\begin{tabular}{|c|c|c|c|}
\hline Group characteristics & Intervention & Control & $\mathbf{p}$ \\
\hline \multicolumn{4}{|l|}{ Gender } \\
\hline Male & $20(57.1)$ & $23(65.7)$ & \multirow[t]{2}{*}{0.46} \\
\hline Female & 15 (42.9) & $12(34.3)$ & \\
\hline \multicolumn{3}{|l|}{ Occupation } & \multirow[t]{6}{*}{0.71} \\
\hline Unemployed & $10(28.6)$ & $13(37.1)$ & \\
\hline Homemaker & $15(42.9)$ & $13(27.1)$ & \\
\hline Worker & $1(2.9)$ & $0(0.0)$ & \\
\hline Retired & $2(5.7)$ & $4(11.4)$ & \\
\hline Self-employed & $7(20.0)$ & $5(14.3)$ & \\
\hline \multicolumn{3}{|l|}{ Income } & \multirow{4}{*}{0.20} \\
\hline Suffices & $4(11.4)$ & $8(22.9)$ & \\
\hline Somehow suffices & $11(31.4)$ & $14(40.0)$ & \\
\hline Doesn't suffice & $20(57.1)$ & $13(27.1)$ & \\
\hline \multicolumn{3}{|l|}{ Location } & \multirow[t]{3}{*}{1} \\
\hline City & $21(60.0)$ & $21(60.0)$ & \\
\hline Village & $14(40.0)$ & $14(40.0)$ & \\
\hline \multicolumn{3}{|l|}{ Education } & \multirow[t]{4}{*}{0.40} \\
\hline Diploma & $31(88.6)$ & 27 (77.1) & \\
\hline Below diploma & $3(8.6)$ & $7(20.0)$ & \\
\hline Academic & $1(2.9)$ & $1(2.9)$ & \\
\hline \multicolumn{3}{|l|}{ Insurance status } & \multirow[t]{3}{*}{0.17} \\
\hline Yes & $28(80.0)$ & $23(65.7)$ & \\
\hline No & $7(20.0)$ & $12(34.2)$ & \\
\hline \multicolumn{3}{|l|}{ Marital status } & \multirow[t]{5}{*}{0.28} \\
\hline Married & $31(88.6)$ & $26(74.3)$ & \\
\hline Single & $3(8.6)$ & $4(11.4)$ & \\
\hline Spouse died & $1(2.9)$ & $4(11.4)$ & \\
\hline Separated & $0(0.0)$ & $1(2.9)$ & \\
\hline \multicolumn{3}{|l|}{ Living with } & \multirow[t]{4}{*}{0.49} \\
\hline Alone & $0(0.0)$ & $2(5.7)$ & \\
\hline Spouse and children & $32(91.4)$ & $28(80.0)$ & \\
\hline Parents & $3(8.6)$ & $5(14.3)$ & \\
\hline \multicolumn{4}{|l|}{ Caregiver } \\
\hline Spouse & $23(65.7)$ & $31(88.6)$ & 0.04 \\
\hline Children & $24(68.6)$ & $22(62.9)$ & 0.80 \\
\hline Parents & $6(17.1)$ & $6(17.1)$ & 1 \\
\hline Patient & $4(11.4)$ & $1(2.9)$ & 0.35 \\
\hline
\end{tabular}

Data are expressed as number (percentage). $\mathrm{p}$ values calculated by Chi-square, Fisher's exact test control group (62.9\%). Furthermore, the level of adherence to fluid intake restriction before intervention was poor for most participants in the intervention group (88.6\%) and the control group (94.3\%). Table 2 shows that all participants in this study had poor treatment adherence $(<50 \%)$ in one of the components. However, the adherence to medication regimen before the intervention was good for most patients in the intervention group (60\%) and control group (77\%). This reflects the higher importance given to adherence to medication regimen by patients.

The results showed that most of the barriers to medication regimen adherence (medication regimen and food and fluid intake restrictions) for the participant sample included lack of knowledge about the right amount of daily fluid intake (90\%) and forgetting to take medications due to multiplicity of drugs (80\%) and lack of belief in the impact of diet adherence on treatment (60\%).

\section{DISCUSSION}

Intervention method which pays attention to self-efficacy comprises sixstep method and motivational interviewing. In the six-step method, not only interview but also self-monitoring like keeping diary is adopted to improve patient's awareness. In all steps, verbal persuasion is provided. Step 2 includes vicarious experiences by providing stories about other patients. Steps 4 and 5 include performance accomplishments. In the literature review, no study with similar results in this area was found. Kakudate et al. (2009) investigated a cognitive behavioral approach for oral hygiene instruction. The purpose of the study was to determine whether a six-step behavioral cognitive method is more effective than traditional oral hygiene instruction. Data analysis showed that the intervention group had a significantly higher self-efficacy than those of the control group [15]. The results of the present study showed that cognitive behavioral intervention is an effective method to improve the score of adherence to medication regimen in patients undergoing hemodialysis. Score increase in adherence to medication regimen in patients of the intervention group in this study can be the nature of multilateral, cognitive behavioral intervention. Cognitive behavioral therapy can help patients to find out their problems and change them. The result of these changes can cause the improvement in person's emotion and behavior. Cognitive behavioral approach is useful for patients who have multiple disorders. It also can be helpful for people who don't have any belief about compliance with their therapeutic diet [12]. In the first step of cognitive behavioral intervention, the causes of failure in compliance are identified and adherence barriers are examined. The patient problem in compliance with medication regimen is investigated through a face to face interview. These measures can be effective in identifying and removing barriers of noncompliance. Results of the systematic study of Matteson and Russell, aimed to investigate the interventions to improve adherence of therapeutic diet

Table 2: Distribution of relative and absolute adherence to diet, medication, and restricted fluid intake before the intervention for the intervention and control groups

\begin{tabular}{llll}
\hline Treatment program & Time & Before intervention & Control \\
\cline { 2 - 4 } & Adherence rate & Intervention & $22(62.9)$ \\
\hline Diet adherence & Poor $(<50 \%)$ & $29(83.9)$ & $13(37.1)$ \\
& Relatively favorable $(75-50 \%)$ & $6(17.1)$ & $0(0.0)$ \\
Fluid restriction adherence & Good $(>75 \%)$ & $0(0.0)$ & $35(100.0)$ \\
& Total & $35(100.0)$ & $33(94.3)$ \\
& Poor $(<50 \%)$ & $31(88.6)$ & $2(5.7)$ \\
Medication adherence & Relatively favorable $(75-50 \%)$ & $4(11.4)$ & $0(0.0)$ \\
& Good $(>75 \%)$ & $0(0.0)$ & $35(100.0)$ \\
& Total & $35(100.0)$ & $1(2.9)$ \\
& Poor $(<50 \%)$ & $3(8.6)$ & $7(20.0)$ \\
& Relatively favorable $(75-50 \%)$ & $11(31.4)$ & $27(77.0)$ \\
& Good $(>75 \%)$ & $21(60.0)$ & $35(100.0)$ \\
\hline
\end{tabular}

Data are expressed as number (percentage) 
Table 3: The impact of cognitive behavioral intervention on removing barriers to treatment adherence in intervention and control groups before and after intervention

\begin{tabular}{|c|c|c|c|c|c|}
\hline \multirow[t]{2}{*}{ Treatment Program } & \multirow{2}{*}{$\begin{array}{l}\text { Time } \\
\text { Removal rate }\end{array}$} & \multicolumn{2}{|c|}{ Before intervention } & \multicolumn{2}{|c|}{ After intervention } \\
\hline & & Intervention & Control & Intervention & Control \\
\hline \multirow[t]{5}{*}{ Barriers to treatment adherence } & Complete removal $(<50 \%)$ & $0(0.0)$ & $0(0.0)$ & $32(49.1)$ & $3(9.4)$ \\
\hline & Partial removal (75-50\%) & $13(37.1)$ & $16(45.7)$ & $1(2.9)$ & $15(46.9)$ \\
\hline & No removal (>75\%) & $22(62.9)$ & $19(54.3)$ & $1(2.9)$ & $14(43.8)$ \\
\hline & Mean & $46.5 \pm 6.68$ & $44.4 \pm 5.98$ & $13.6 \pm 7.70$ & $40.9 \pm 8.46$ \\
\hline & $\mathrm{p}$-value & 0.15 & & $<0.001$ & \\
\hline
\end{tabular}

Data expressed as number (percent); mean \pm SD. p-values calculated by Independent t-test results

in chronic patients, showed that cognitive behavioral interventions are more effective than other interventions that have been carried out in adherence of therapeutic diet [16]. The results of the present study also confirmed that to improve medication adherence, first it is necessary to establish effective communication with the patient treatment, identify the reasons and barriers of non-adherence in patient and increase patient motivation to change behavior. The study results of Baumann and Dang entitled helping patients with chronic disease to improve adherence and overcome self-care obstacles showed that the first step to help hemodialysis patients in self-care program (including medication adherence) is to identify patient's problem and investigate the reasons for non-adherence. The authors acknowledge that such strategies to overcome self-care barriers are motivational and cognitive interview of individual beliefs and increased social support [8]. The results confirmed the effectiveness of these interventions compared with other training methods. Kara et al. (2007) carried out a study entitled, "nonadherence with diet and fluid restrictions and perceived social support in patients receiving hemodialysis." Their results showed that more than $50 \%$ of hemodialysis patients showed non-adherence with diet and fluid restrictions. In the current study, the results of the rate of treatment adherence show that all individuals participating in the study had poor adherence on one adherence component. Browne and Merighi (2010) in a systematic study entitled, "barriers to adult hemodialysis patients' self-management of oral medications" that include pill burden, demographic and socioeconomic variables, psychosocial factors, health literacy, patient satisfaction, and health beliefs. In the current study, responses given to the TABQs show that forgetting to get recommended medicines and foods is one of the greatest barriers to medication adherence which is consistent with the results of research conducted by other researchers [5].

The results of this study showed that cognitive behavioral intervention can lead to improvement in adherence to treatment regimen in hemodialysis patients. Therefore, it is suggested the effect of this kind of intervention be investigated in other chronic diseases. Because, in this study, the results of the intervention were assessed by self-report and through questionnaires, it is suggested that the effect of intervention also be performed by examining the results of laboratory tests of patients.

\section{Limitations of research}

Removing barriers to adherence due to economic difficulties could not be investigated by the researcher and was one of the limitations of this study. The participants' mental condition when completing the questionnaire could affect their responses, but could not be controlled by the researcher. However, the researcher tried to collect data at the right time and place.

\section{CONCLUSION}

The overall results of the study showed that cognitive behavioral intervention removes treatment adherence barriers in hemodialysis patients and can be used to improve treatment adherence in these patients. It is suggested that this type of intervention be also used in other chronic diseases.

\section{ACKNOWLEDGMENTS}

This article is the result of a research project approved by the Tehran University of Medical Sciences with registration number 91-0428-20321. The study was also registered at the Iranian Registry of Clinical Trials with registration number IRCT201211101599N21. The researcher warmly appreciates the staff of hemodialysis wards of Imam Reza Hospital of Lar and Vali-e-Asr Hospital of Lamerd and all respected patients who worked with us in this study.

\section{REFERENCES}

1. Chauveau P, Nguyen H, Combe C, Chêne G, Azar R, Cano N, et al. Dialyzer membrane permeability and survival in hemodialysis patients. Am J Kidney Dis 2005;45(3):565-71.

2. Moneret-Vautrin DA, Morisset M, Flabbee J, Beaudouin E, Kanny G. Epidemiology of life-threatening and lethal anaphylaxis: A review. Allergy 2005;60(4):443-51.

3. Cinar S, Barlas GU, Alpar SE. Stressors and coping strategies in hemodialysis patients. Pak J Med Sci 2009;25(3):447-52.

4. Denhaerynck K, Manhaeve D, Dobbels F, Garzoni D, Nolte C, De Geest S. Prevalence and consequences of nonadherence to hemodialysis regimens. Am J Crit Care 2007;16(3):222-35.

5. Browne T, Merighi JR. Barriers to adult hemodialysis patients' self-management of oral medications. Am J Kidney Dis 2010;56(3):547-57.

6. Kara B, Caglar K, Kilic S. Nonadherence with diet and fluid restrictions and perceived social support in patients receiving hemodialysis. J Nurs Scholarsh 2007;39(3):243-8.

7. Welch JL, Siek KA, Connelly KH, Astroth KS, McManus MS, Scott L, et al. Merging health literacy with computer technology: Self-managing diet and fluid intake among adult hemodialysis patients. Patient Educ Couns 2010;79(2):192-8.

8. Baumann LC, Dang TT. Helping patients with chronic conditions overcome barriers to self-care. Nurse Pract 2012;37(3):32-8.

9. Safari M, Shojaizadeh D, Ghofranifard F, Haidarian A, Pakpor-hajaghaA. Models and methods of health education and health promotion. 3rd ed. Tehran, Iran: Asar Sobhan Publication; 2009. p. 23-4.

10. Sathvik BS, Narahari MG, Gurudev KC, Parthasarathi G. Impact of clinical pharmacist-provided education on medication adherence behaviour in ESRD patients on haemodialysis. Iran J Pharm Sci 2009;5(1):21-30.

11. Beraz S, Mohammadi E, Boromand B. The effect of dietary regimen education on the laboratory variables and interdialytic weight gain in hemodialysis patients. J Shahrekord Univ Med Sci 2006;8(1):20-7.

12. Sharp J, Wild MR, Gumley AI. A systematic review of psychological interventions for the treatment of nonadherence to fluid-intake restrictions in people receiving hemodialysis. Am J Kidney Dis 2005;45(1):15-27.

13. Sharp J, Wild MR, Gumley AI, Deighan CJ. A cognitive behavioral group approach to enhance adherence to hemodialysis fluid restrictions: A randomized controlled trial. Am J Kidney Dis 2005;45(6):1046-57.

14. Albright $\mathrm{C}$, Farquhar J. Introduction to clinical medicine. In: 
Greene HM, editor. Principles of Behavioral Change. Philadelphia, PA BC Decker, Inc.; 1996. p. 762-7.

15. Kakudate N, Morita M, Sugai M, Kawanami M. Systematic cognitive behavioral approach for oral hygiene instruction: A short-term study.
Patient Educ Couns 2009;74(2):191-6.

16. Matteson ML, Russell C. Interventions to improve hemodialysis adherence: A systematic review of randomized-controlled trials. Hemodial Int 2010;14(4):370-82. 